\title{
NEW MOLECULAR MARKERS INVOLVED IN IMMUNE SYSTEM HOMEOSTASIS AND HEMOPOIETIC ORGAN DEVELOPMENT ARE DIFFERENTIALLY REGULATED DURING OOCYTES IN VITRO MATURATION
}

Lisa Moncrieff',2, Ievgeniia Kocherova ${ }^{3}$, Artur Bryja ${ }^{3}$, Wiesława Kranc ${ }^{3}$, Joanna Perek ${ }^{3}$, Piotr Celichowski², Magdalena Kulus ${ }^{4}$, Bartosz Kempisty ${ }^{2,3,4,5}$, Paul Mozdziak ${ }^{6}$ Michal Jeseta $^{5}$

\begin{abstract}
The growth and maturation of the oocyte is a dynamic process which requires a variable supply of hormones, growth factors and energy. These needs are met partially by the surrounding somatic cells and the cumulus-oocyte complex, which communicate bi-directionally via gap junctions. Identifying and analyzing protein expression in the oocyte can provide insight in its development and growth. Further, like bone marrow stem cells, if relevant marker genes are found in oocytes, there is a potential for the oocyte to be manipulated into becoming hemopoietic stem cells. In this study, porcine oocytes were isolated and subjected to microarray analysis to compare the oocyte gene expression in vivo and in vitro maturation (IVM). Genes identified belonged to both 'hemopoietic or lymphoid organ development'(G0:0048534) and 'immune system development' (GO:0002520), and the markers can be used to identify several activities such as cell migration, neurogenesis and proliferation. The following are the identified genes and all were downregulated after IVM to varying degrees: ID2, VEGFA, TGFBR3, INHBA, CDK6, BCL11A, MYO1E, ITGB1, EGR1, NOTCH2, SPTA1, KIT and TPD52. Our results should provide new markers to further investigate oocyte development and growth regulation.
\end{abstract}

Running title: Markers of hemopoietic organ development

Keywords: pig, oocyte, microarray, IVM

\footnotetext{
${ }^{1}$ School of Medicine, Medical Sciences and Nutrition, University of Aberdeen, Aberdeen, United Kingdom

${ }^{2}$ Department of Histology and Embryology, Poznań University of Medical Sciences, Poznań, Poland

${ }^{3}$ Department of Anatomy, Poznan University of Medical Sciences, Poznań, Poland

${ }^{4}$ Department of Veterinary Surgery, Nicolaus Copernicus University in Toruń, Toruń, Poland

${ }^{5}$ Department of Obstetrics and Gynecology, University Hospital and Masaryk University, Brno, Czech Republic

${ }^{6}$ Graduate Physiology Program, North Carolina State University, Raleigh, USA

* Correspondence: bkempisty@ump.edu.pl

Full list of author information is available at the end of article
} 


\section{Introduction}

The long process of creating a viable female gamete for reproduction involves the growth and maturation of the oocyte. It is critical that the needs of the oocyte is met, such as receiving hormones, growth factors or energy, so that folliculogenesis and oogenesis can successfully occur [1]. Due to the dynamic requirements of the oocyte during its development, gene expression will change between the transition of in vivo and in vitro maturation (IVM) which could give information on how the gamete cells interacts with its surrounding somatic cells [2].

The spheroid structure of the ovarian follicle consists of an oocyte, layers of two cell population of granulosa cells called cumulus cells (CCs) and mural granulosa cells (GCs), and also theca cells. Granulosa cells and theca cells release hormones that stimulate essential morphological and metabolic changes in the oocyte [3]. Increasing evidence shows that the quality and capability of gamete cells, to achieve successful development, ovulation and fertilization, are dependent on how it interacts with the somatic cells and their extracellular relationship [4]. CCs which directly surround the oocyte are connected via gap junctions (made up of connexons), and have bi-directional communication which exchanges proteins [5]. This specific relationship is called the cumulus-oocyte complex (COC).

Bone marrow stem cells have been successfully influenced to differentiate into human embryonic progenitor cells which can then further develop into specialized hematopoietic cells such as erythrocytes or leucocytes [6]. This was accomplished through identifying a h-ESC marker (CD34+) and applying an appropriate culture medium to induce hemopoiesis. In a similar approach, identifying marker genes for hemopoietic or lymphoid organ development in oocytes could make them a new source of hematopoietic stem cells [7]. Furthermore, more could be learnt about oocyte regulation, proliferation and development by identifying biomarkers related to immune system development [8].

To study protein synthesis and identify biomarkers for hemopoiesis or immune system development in a porcine oocyte model, microarray techniques can be used [9].

Oocytes from pubertal crossbred landrace gilts were isolated. Then the protein expression of genes belonging to GO BP ontology terms 'hemopoietic or lymphoid organ development' or 'immune system development' was selected and analyzed for comparison of in vivo and in vitro maturation expressions. These results provide insight into the development, growth and differentiation capability of the oocyte.

\section{Material and methods Experimental design}

Oocytes were collected and subjected to two Brilliant Cresyl Blue (BCB) tests and divided into two groups. The first group ("before IVM") included oocytes graded as $\mathrm{BCB}$-positive $\left(\mathrm{BCB}^{+}\right)$and directly exposed to microarray assay. The second group ("after IVM") included $\mathrm{BCB}^{+}$oocytes which were then in vitro matured, and if classified as $\mathrm{BCB}^{+}$in second BCB test passed to molecular analyses.

\section{Animals}

A total of 45 pubertal crossbred Landrace gilts bred on a commercial local farm were used in this study. They had a mean age of 155 days (range $140-$ 170 days) and a mean weight of $100 \mathrm{~kg}$ (95-120 kg). All animals were bred under the same conditions and fed the same forage (depending on age and reproductive status). All experiments were approved by the Local Ethic Committee (approval no. 32/2012).

\section{Collection of porcine ovaries and cumulus- oocyte-complexes (COCs)}

The ovaries and reproductive tracts were recovered at slaughter and transported to the laboratory within $40 \mathrm{~min}$. at $38^{\circ} \mathrm{C}$ in $0.9 \% \mathrm{NaCl}$. To provide optimal conditions for subsequent oocyte maturation and fertilization in vitro, the ovaries of each animal were placed in a 5\% fetal bovine serum solution (FBS; Sigma-Aldrich Co., St. Louis, MO, USA) in PBS. Single large follicles $(>5 \mathrm{~mm})$ were opened by puncturing with a $5 \mathrm{ml}$ syringe and $20-\mathrm{G}$ needle in a sterile Petri dish, and COCs were recovered. The COCs were washed three times in modified PBS supplemented with $36 \mu \mathrm{g} / \mathrm{ml}$ pyruvate, $50 \mu \mathrm{g} / \mathrm{ml}$ gentamycine, and $0.5 \mathrm{mg} / \mathrm{ml}$ BSA (Sigma-Aldrich, St. Louis, MO, USA). The COCs were selected under an inverted microscope Zeiss, Axiovert 35 (Lübeck, Germany), counted, and morphologically evaluated. Only COCs of grade I possessing homogeneous ooplasm and uniform, compact cumulus cells were considered for further use, resulting in a total of 300 grade I oocytes ( $3 \times \mathrm{n}=50$ "before IVM" group, 3 $\mathrm{x} \mathrm{n=50} \mathrm{"after} \mathrm{IVM"} \mathrm{group).}$

\section{Assessment of oocyte developmental competence by BCB test}

Brilliant Cresyl Blue (BCB) test was used for assessment of porcine oocytes' quality and maturity. The glucose-6-phosphate (G6PDH) enzyme converts BCB stain from blue to colorless. In oocytes that completed the growth activity of the enzyme decreases and the stain cannot be reduced, resulting in blue oocytes $\left(\mathrm{BCB}^{+}\right)$. To perform the $\mathrm{BCB}$ staining test, oocytes were washed twice in modified Dulbecco's Phosphate Buffered Saline (DPBS) commercially supplemented with $0.9 \mathrm{mM}$ calcium, $0.49 \mathrm{mM}$ magnesium, $0.33 \mathrm{mM}$ pyruvate, and $5.5 \mathrm{mM}$ glucose (Sigma-Aldrich, St. Louis, MO, USA), and additionally with $50 \mathrm{IU} / \mathrm{ml}$ penicillin, $50 \mu \mathrm{g} / \mathrm{ml}$ streptomycin (Sigma-Aldrich, St. Louis, MO, USA), and 0.4\% Bovine Serum Albumin (BSA) [w/v] (Sigma-Aldrich, St. Louis, MO, USA). They were then treated with $13 \mu \mathrm{M}$ 
BCB (Sigma-Aldrich, St. Louis, MO) diluted in DPBS at $38.5^{\circ} \mathrm{C}, 5 \% \mathrm{CO}_{2}$ for $90 \mathrm{~min}$. After treatment, the oocytes were transferred to DPBS and washed twice. During washing, the oocytes were examined under an inverted microscope and classified as stained blue $\left(\mathrm{BCB}^{+}\right)$or colorless $\left(\mathrm{BCB}^{-}\right)$. Only the granulosa cell-free $\mathrm{BCB}^{+}$oocytes were used for subsequent molecular analyses ("before IVM" group) or IVM followed by second BCB test and molecular analyses ("after IVM" group).

\section{In vitro maturation of porcine cumulus-oocyte- complexes (COCs)}

After the first $\mathrm{BCB}$ test, the $\mathrm{BCB}^{+} \mathrm{COC}$ were subjected to IVM. The COCs were cultured in Nunc$\operatorname{lon}^{\mathrm{TM}} \Delta$ 4-well dishes (Thermo Fisher Scientific, Waltham, MA, USA) in $500 \mu \mathrm{l}$ standard porcine IVM culture medium: TCM-199 (tissue culture medium) with Earle's salts and L-glutamine (Gibco BRL Life Technologies, Grand Island, NY, USA), supplemented with $2.2 \mathrm{mg} / \mathrm{ml}$ sodium bicarbonate (Nacalai Tesque, Inc., Kyoto, Japan), $0.1 \mathrm{mg} / \mathrm{ml}$ sodium pyruvate (Sigma-Aldrich, St. Louis, MO, USA), $10 \mathrm{mg} /$ ml BSA (Bovine Serum Albumin) (Sigma-Aldrich, St. Louis, MO, USA), $0.1 \mathrm{mg} / \mathrm{ml}$ cysteine (Sigma-Aldrich, St. Louis, MO, USA), 10\% (v/v) filtered porcine follicular fluid, and gonadotropin supplements at final concentrations of $2.5 \mathrm{IU} / \mathrm{ml}$ hCG (human Chorionic Gonadotropin) (Ayerst Laboratories, Inc., Philadelphia, PA, USA) and $2.5 \mathrm{IU} / \mathrm{ml}$ eCG (equine Chorionic Gonadotropin) (Intervet, Whitby, ON, Canada). Wells were covered with mineral oil overlay and cultured at $38^{\circ} \mathrm{C}$ under $5 \% \mathrm{CO}_{2}$ in air for $22 \mathrm{~h}$, and then for additional $22 \mathrm{~h}$ in medium without hor-

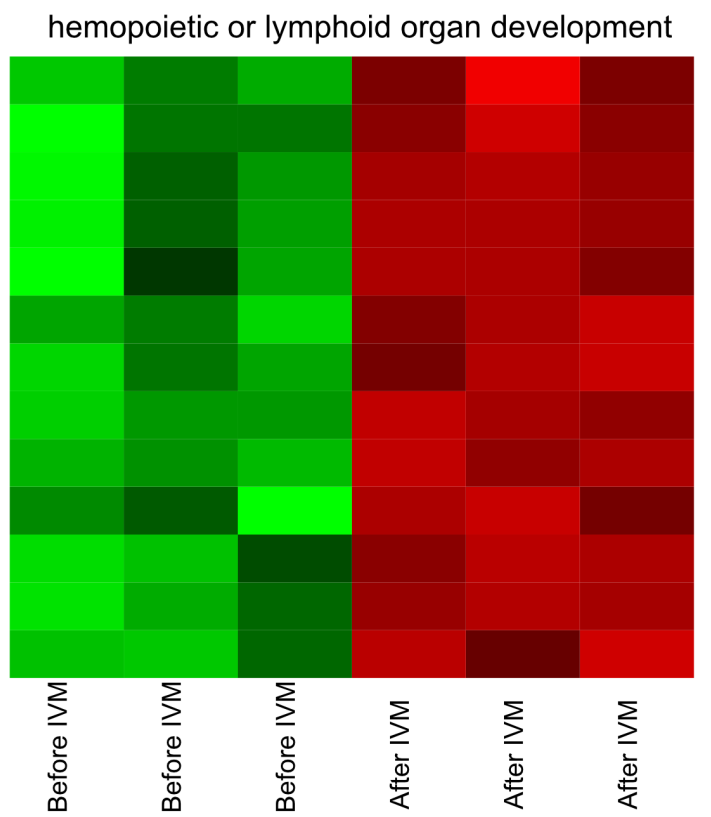

mones. After cultivation, the second BCB staining test was performed, and $\mathrm{BCB}^{+}$oocytes were used for further molecular analyses.

\section{Microarray expression analysis and statistics}

Experiments were performed in three replicates. Total RNA (100 ng) from each pooled sample was subjected to two round sense cDNA amplification (Ambion® WT Expression Kit). The obtained cDNA was used for biotin labeling and fragmentation by Affymetrix GeneChip $₫$ WT Terminal Labeling and Hybridization (Affymetrix). Biotin-labeled fragments of cDNA $(5.5 \mu \mathrm{g})$ were hybridized to Affymetrix $®$ Porcine Gene 1.1 ST Array Strip $\left(48^{\circ} \mathrm{C} / 20\right.$ h). Then, microarrays were washed and stained according to the technical protocol, using Affymetrix GeneAtlas Fluidics Station. The array strips were scanned employing Imaging Station of GeneAtlas System. The preliminary analysis of the scanned chips was performed using Affymetrix GeneAtlasTM Operating Software. Quality of gene expression data was checked according to quality control criteria provided by the software. Obtained CEL files were imported into downstream data analysis software.

All analyzes were performed using BioConductor software, based on the statistical R programming language. For background correction, normalization and summation of raw data, the Robust Multiarray Averaging (RMA) algorithm implemented in "affy" package of BioConductor was applied. Biological annotation was taken from BioConductor "oligo" package where annotated data frame object was merged with normalized data set, leading to a complete gene data table. Statistical significance of

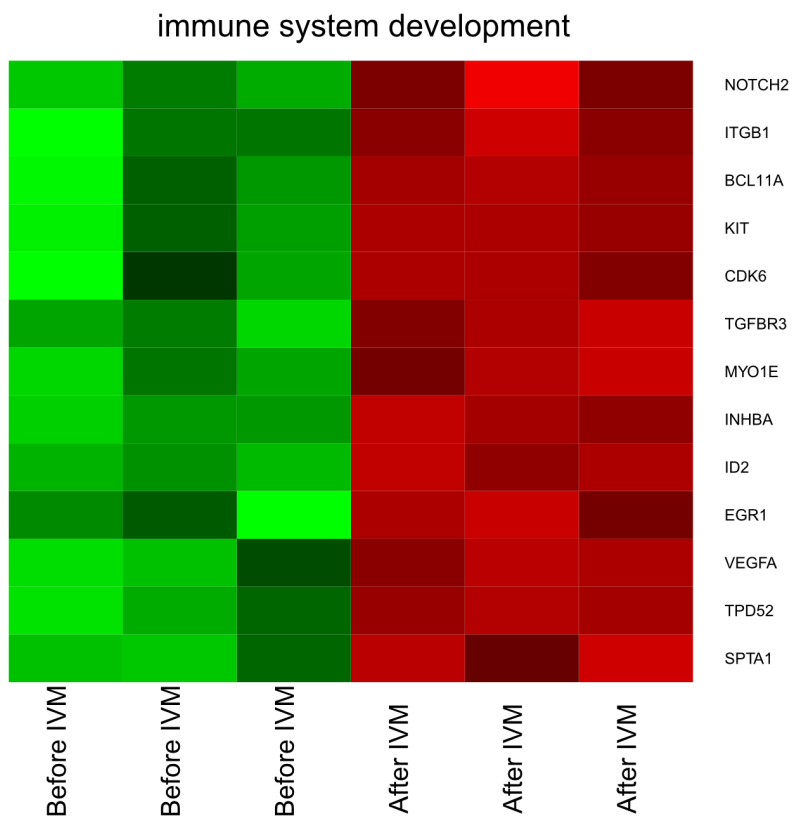

FIGURE 1 Heat map representations of differentially expressed genes belonging to the "hemopoietic or lymphoid organ development" and "immune system development" GO BP terms. Arbitrary signal intensity acquired from microarray analysis is represented by colours (green, higher; red, lower expression). Log2 signal intensity values for any single gene were resized to Row Z-Score scale (from -2 , the lowest expression to +2 , the highest expression for single gene) 
analyzed genes was performed by moderated t-statistics from the empirical Bayes method. Obtained $p$ value was corrected for multiple comparisons using the Benjamini and Hochberg's false discovery rate. The selection of significantly changed gene expression was based on $p$ value beneath 0.05 and expression fold higher than $|2|$.

Functional annotation clustering of differentially expressed genes was performed using DAVID (Database for Annotation, Visualization and Integrated Discovery). Gene symbols for up- or down-regulated genes from each of the compared groups were loaded to DAVID by "RDAVIDWebService" BioConductor package. For further analysis we have chosen the enriched GO terms which has at least 5 genes and $\mathrm{p}$ value (Benjamini) lower than 0.05 . The enriched GO terms were subjected to hierarchical clusterization algorithm and presented as heat maps.

Subsequently we analyzed the relation between the genes belonging to chosen GO terms with GOplot package. The GOplot package had calculated the z-score: the number of up- regulated genes minus the number of down- regulated genes divided by the square root of the count. This information allowed estimating the change course of each gene-ontology term.

Interactions between chosen differentially expressed genes/proteins belonging to ontology group were investigated by STRING10 software (Search Tool for the Retrieval of Interacting Genes). List of gene names were used as query for interaction prediction. Searching criteria based on co-occurrences of genes/proteins in scientific texts (text mining), co-expression and experimentally observed interactions. The results of such analysis generated gene/protein interaction network where the intensity of the edges reflects the strength of interaction score. Besides interaction prediction, STRING also allowed us to perform functional enrichments of GO terms based on previously uploaded gene sets.

\section{Ethical approval}

The research related to animal use has been complied with all the relevant national regulations and instructional policies for the care and use of animals. Bioethical Committee approval no. 32/2012 from 30.06.2012.

\section{Results}

Whole transcriptome profiling by Affymetrix microarray allowed us to analyze the gene expression changes in freshly isolated oocytes, before in vitro procedure ("before IVM"), in relation to after in vitro maturation ("after IVM"). By Affymetrix® Porcine Gene 1.1 ST Array we have examined expression of 12258 porcine transcripts. Genes with fold change higher than $|2|$ and with corrected $p$ value lower than 0.05 were considered as differentially expressed. This set of genes consisted of 419 different transcripts. Subsequently, the genes were used for identification of significantly enriched GO BP terms.

DAVID (Database for Annotation, Visualization and Integrated Discovery) software was used for extraction of the genes belonging to regulation of "hemopoietic or lymphoid organ development" and "immune system development" gene ontology Biological Process term (GO BP). We found that 13 genes from these GO BP term were significantly represented in down-regulated gene sets. This set of genes was subjected to hierarchical clusterization procedure and presented as heat maps (Fig. 1).

Set of the differentially expressed genes belonging to "hemopoietic or lymphoid organ development" and "immune system development" GO BP terms

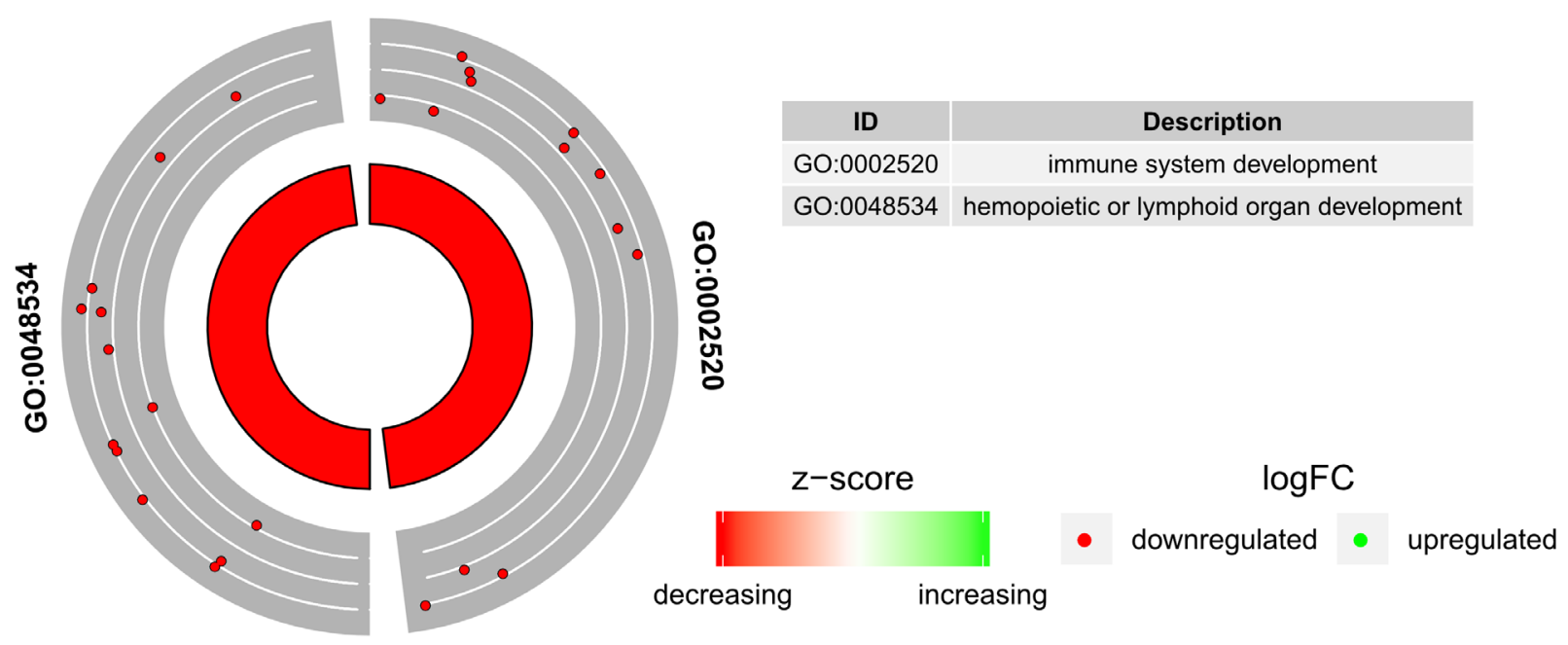

FIGURE 2 The circle plot showing the differently expressed genes and z-score "hemopoietic or lymphoid organ development" and "immune system development" GO BP Terms. The outer circle shows a scatter plot for each term of the fold change of the assigned genes. Red circles display down- regulation and green ones up- regulation. The inner circle shows the z-score of each GO BP term. The width of each bar corresponds to the number of genes within GO BP term and the color corresponds to the z-score 


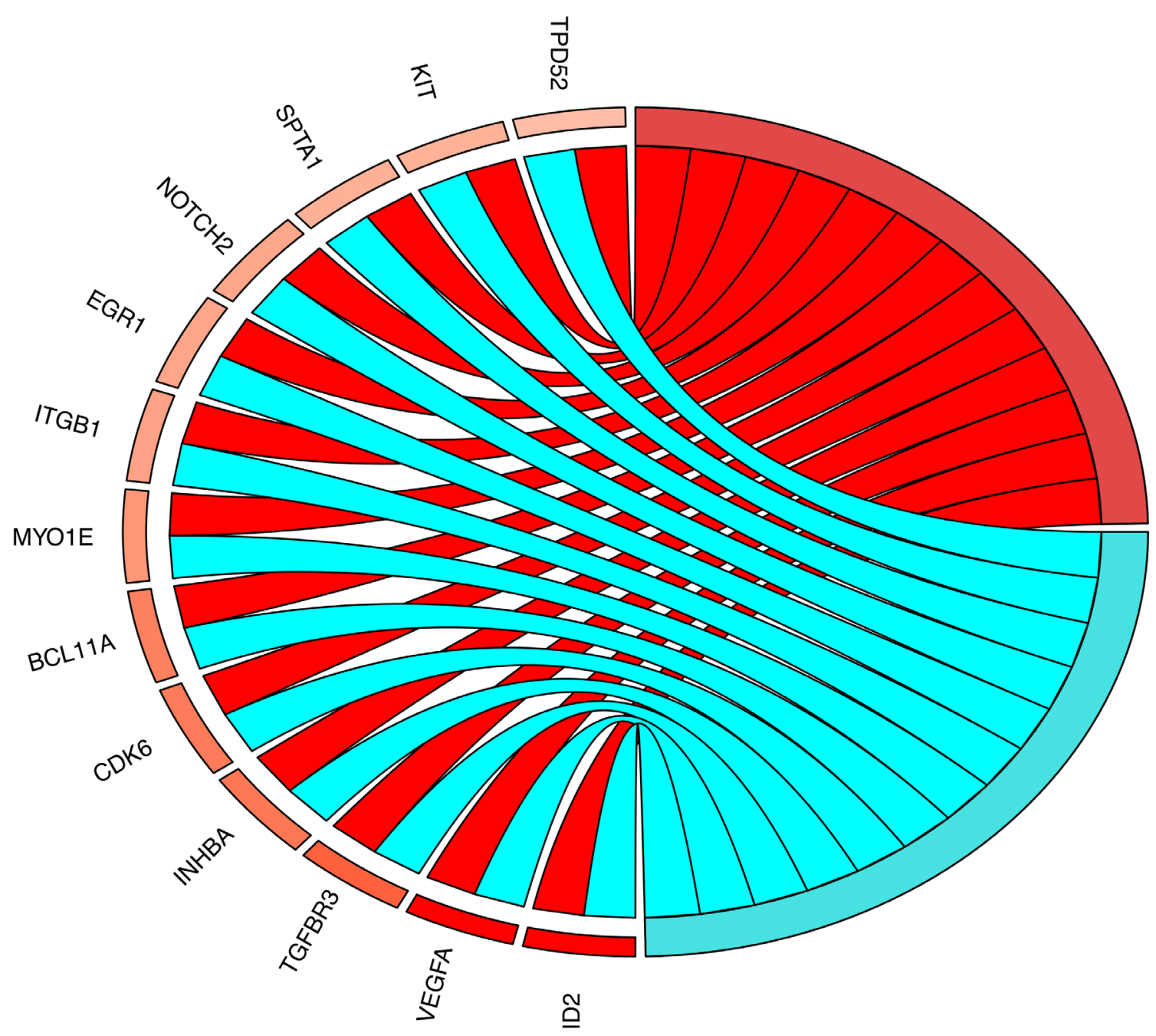

GO Terms

immune system development

hemopoietic or lymphoid organ development

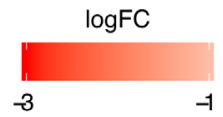

FIGURE 3 The representation of the mutual relationship between differently expressed genes that belongs to "hemopoietic or lymphoid organ development" and "immune system development" GO BP Terms. The ribbons indicate which gene belongs to which categories. The middle circle represents logarithm from fold change (LogFC) between before IVM and after IVM respectively. The genes were sorted by logFC from most to least changed gene

with their official gene symbols, ratio, Entrez Gene IDs and corrected p values were shown in table $\mathbf{1}$.

The enrichment of each GO BP term as well KEGG pathway were calculated as z-score and shown on the circle diagram (Fig. 2)

Moreover, in Gene Ontology database genes that formed one particular GO group can also belong to other different GO term categories. By this reason we explore the gene intersections between selected GO BP terms. The relation between those GO BP terms was presented as circle plot (Fig. 3) as well as heatmap.

STRING-generated interaction network was created with differentially expressed genes belonging to the "fatty acid metabolic process" ontology group. The intensity of the edges reflects the strength of interaction score (Fig. 4).

\section{Discussion}

In our study, a group of 13 genes expressed in our porcine oocyte model were selected and analyzed: ID2, VEGFA, TGFBR3, INHBA, CDK6, BCL11A, MYO1E, ITGB1, EGR1, NOTCH2, SPTA1, KIT and TPD52. All genes belonged to both ontology groups which are 'hemopoietic or lymphoid organ development' and 'immune system development'. Furthermore, they were all downregulated after IVM compared to before. This provides an indication of the cell type's potential to differentiate into hemopoietic or lymphoid cell lines $[10,11]$.

The most downregulated gene after IVM was found to be ID2. The differentiation of $\mathrm{CD} 4^{+} \mathrm{T}$ cells, and their regulatory $\mathrm{T}\left(\mathrm{T}_{\text {reg }}\right)$ population which are responsible for immune homeostasis, is regulated 


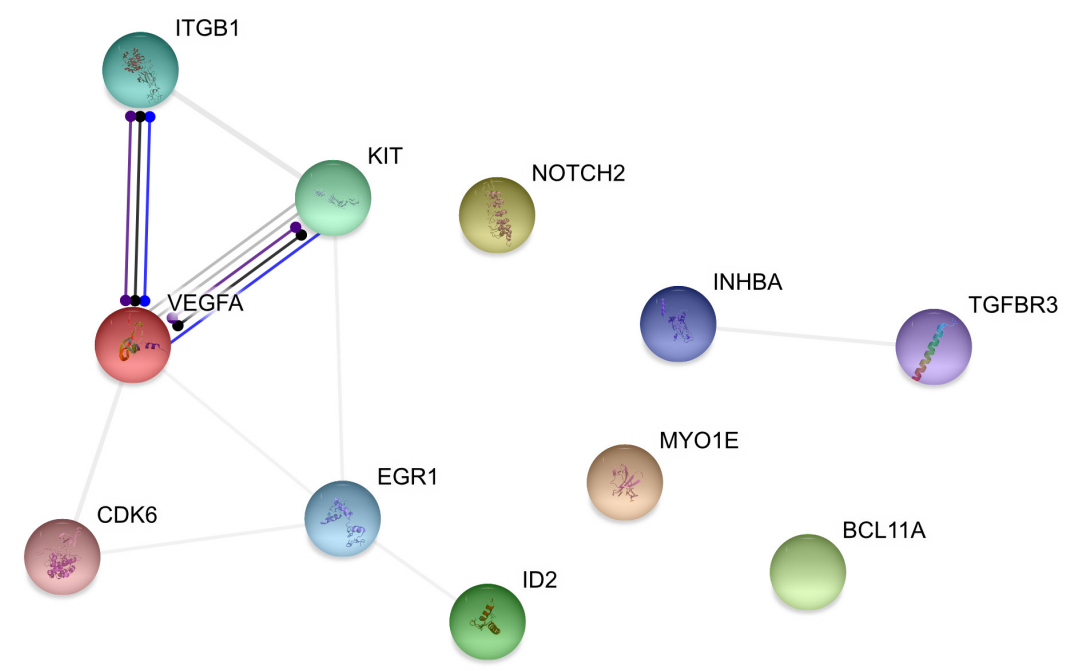

\section{Action Types}

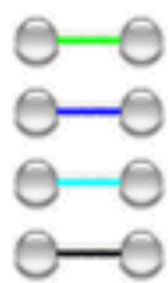

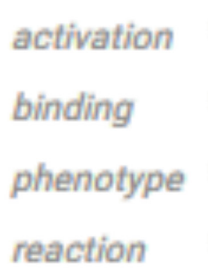

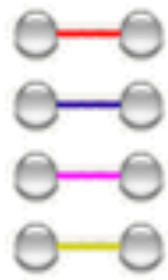

inhibition

catalysis

posttranslational modification

transcriptional regulation
Action effects

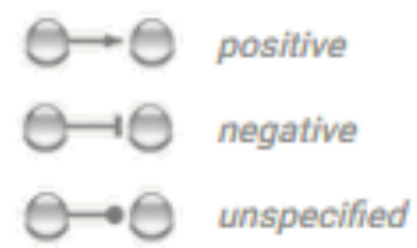

FIGURE 4 STRING-generated interaction network between genes that belongs to the "hemopoietic or lymphoid organ development" and "immune system development" GO BP Terms. The intensity of the edges reflects the strength of interaction score

partly by Id2 (inhibitor of DNA binding 2) and its family member Id3 [12,13]. The Id proteins (1-4) in general control lymphocyte development and homeostasis through their inhibitory effect on helix-loop-helix (HLH) DNA transcription factors [14]. Our results for ID2 downregulated expression in the oocyte after IVM is supported by Chermuła et al.'s own study [15], which may indicate a role in the development and maturation of the oocyte.

Vascular endothelial growth factor-A (VEGFA) was the second most downregulated gene in our two ontology groups. This is a well-studied gene for its role in neuron differentiation for vascularization and neurogenesis [16], and from our study is known to bind and show an increase in activity with ITG$B 1$ and KIT (refer to Fig. 4). Inflammation results in increased expression of VEGFA, as the immune system needs blood vessels to form in order to fulfill, nutrient and energy requirements for the inflamed area [17]. In the ovarian follicle, the important process of angiogenesis, which provides energy and promotes cell migration, relies on VEGFA [18]. The observation of its downregulation after the occurrence of IVM is also show by Chermuła et al. [19].

Following VEGFA in the downregulated genes, is transforming growth factor-beta receptor 3 (TGF$B R 3$ ). Its protein TFG $\beta$ (also known as betaglycan), catalyzes endocrine hormones inhibin $\mathrm{A}$ and $\mathrm{B}$ to
TGF $\beta$ whilst also inhibiting the activity of activin and bone morphogenetic proteins (BMPs) [20]. Activin promotes follicle stimulating hormone (FSH) secretion, whilst inhibin does the reverse, and therefore the expression of TGFBR3 must have a clear influence on the growth of the ovarian follicle [21]. Various studies are connecting unusual expression of TFG $\beta$ to the development of cancer and inflammatory disease, as cell migration concerning the immune system and proliferation of cells become unregulated [22,23].

The fourth most downregulated gene was Inhibin- $\beta$ A (INHBA) is known to be expressed in cumulus cells to which it affects oocyte competence and its differential expression is a potential marker for small and dominant granulosa cell follicles [24,25]. Various studies have shown that high expression of INHBA is associated with adverse effects for cancer treatment due to poor immune system response $[26,27]$. This highlights the protective role of $I N$ $H B A^{\prime}$ 's regulation even in oocyte development. Overexpression of cyclin-dependent kinase 6 (CDK6) is also linked to unregulated cell proliferation for the G1/S cell cycle transition, and also by inhibiting miRNA-320d, leading to cancer progression [28,29]. Other CDKs appear to compensate in CDK6 is absent in oocyte development, and the resulting ovary will still be healthy [30]. 
$B C L 11 A$ is the sixth most downregulated gene. The transcription factor is known to be involved in hemopoietic development, specifically in monitoring the expression of fetal hemoglobin (HbF) [31]. Several components on beta globin genes cluster is occupied by $B C L 11 A$; therefore, it is recognized a key therapeutic target for sickle-cell disease [32]. While we have identified a change in expression from before and after IVM, more research should be done to investigate the genes possible effects on oocyte development and growth such as providing increased blood cells and energy to the oocyte.

$M Y O 1 E$ is the seventh most downregulated gene. The adhesion and B cell migration for high end othelial venules incorporate the involvement of Myo1e, and is found to be necessary for the FAK/PI3K/RAC1 signaling pathway [33]. Being specialized capillary blood vessels, high-endothelial venules are responsible for the trafficking of white blood cells [34]. Therefore, MYO1E must have a crucial role in preserving oocyte competence and protecting the cell population. In a study done by Borys et al., MY01E was also found to be downregulated after IVM in oocytes [35].

The eight most downregulated gene is Integrin B-1 (ITGB1). Xu et al. have shown that ITGB1 is a potential therapeutic target because of its effects the cell migration and metastasis of esophageal squamous cell carcinoma (ESCC) [36]. Although more information is needed to know the gene's role in folliculogenesis and oogenesis, ITGB1 is involved in implantation of the ovary and trophoblastic differentiation [37].

Early growth response 1 (EGR1), which encodes a nuclear transcription factor, was the fifth least downregulated gene. Various signaling molecules such as hormones, neurotransmitters, growth and differentiation factors will increase the activity of the gene [38]. Possibly through its interaction with NF- $\kappa$ or inflammatory cytokines, EGR1 has been shown to induce apoptosis for various cell types including granulosa cells during follicular atresia [39]. FSH is also induced by EGR1, an essential gonadotropin for the ovarian follicle suggesting the gene promotes the growth of the oocyte [40].

Another gene we selected and analyzed in our ontology groups is NOTCH2. The expression of NOTCH2 is essential for healthy development of the primordial follicle, according to knockout mice studies of the gene for polycystic ovary syndrome (PCOS) [41]. Our results that NOTCH2 is downregulated after IVM compared to before in oocytes is supported by the results Stefańska et al. [42], suggesting it has a regulatory role in the transition.

The second least downregulated gene is KIT, a cytokine receptor and produces protein members of the tyrosine-kinase family. Cell proliferation of the oocyte and anti-apoptosis of the pre-antral follicle is aided by the phosphorylation from KIT ligands [43]. Furthermore, the protein encoded by KIT can be used as a marker for hematopoietic stem cells [44]. Finally, tumor protein D52 (TPD52) was the least downregulated gene from both ontology groups. Overexpression of TPD52 is linked to increased cell migration and tumor growth $[45,46]$. Not much is known about this gene's effect on the oocyte, and further research should be done.

Through our study, various genes which are present in the oocyte and effect the immune system and hemopoiesis have been analyzed. The expression of these genes suggests the oocyte could have stem cell like qualities in long term in vitro cultures; however, after IVM these are downregulated. This downregulation could be for regulatory purposes during the development and maturation of the oocyte, as these genes influence important cellular activities such as cell migration, neurogenesis and proliferation. Furthermore, the COC will be affected by the change of protein expression in the oocyte and this effect should be studied. New IVM oocyte biomarkers have found from the differential expression of these 13 genes from our microarray approach.

\section{Conclusions}

To summarize, the gene expression of a porcine oocyte model was compared to before and after IVM. Thirteen genes were found to belong to ontological groups 'hemopoietic or lymphoid organ development' (GO:0048534) and 'immune system development' (GO:0002520). Possibly due to their regulatory roles for the developing and mature oocyte, these genes were all downregulated after IVM and could be used as biomarkers. The regulation of genes roles related to' immune system development' has also been emphasized great importance in maintaining homeostasis, preventing cancer aggression and improving treatment resistance. From the effects of cell migration, neurogenesis or proliferation, the proteins encoded have an impact on cell function and competence of the oocyte. Furthermore, the oocyte and its surrounding granulosa cells are indicated to have potential stem cell characteristics for the hematopoietic and lymphoid cell lines which could be further investigated for future studies.

\section{Acknowledgements}

Not applicable.

\section{Corresponding author}

Bartosz Kempisty PhD, Department of Histology and Embryology, Department of Anatomy, Poznan University of Medical Sciences, 6 Święcickiego St., 60-781 Poznań, Poland Tel./Fax: +48 618546418 / +48618546440, e-mail: bkempisty@ump.edu.pl.

\section{Conflict of interest statement}

The authors declare they have no conflict of interest.

\section{References}

1. Gosden R, Lee B. Portrait of an oocyte: Our obscure origin. J Clin Invest. 2010;120:973-83; DOI:10.1172/JCI41294.

2. Eichenlaub-Ritter U, Peschke M. Expression in in-vivo and in-vitro growing and maturing oocytes: focus on regulation of expression at the 
translational level. Hum Reprod Update. 2002;8:21-41; DOI:10.1093/ humupd/8.1.21.

3. Kranc W, Celichowski P, Budna J, Khozmi R, Bryja A, Ciesiółka S, Rybska M, Borys S, Jeseta M, Bukowska D, Antosik P, Brüssow KP, Bruska M, Nowicki M, Zabel M, Kempisty B. Positive regulation of macromolecule metabolic process belongs to the main mechanisms crucial for porcine oocytes maturation. Adv Cell Biol. 2017;5:15-31; DOI:10.1515/ acb-2017-0002.

4. Huang Z, Wells D. The human oocyte and cumulus cells relationship New insights from the cumulus cell transcriptome. Mol Hum Reprod. 2010;16:715-25; DOI:10.1093/molehr/gaq031.

5. Shimada M. Cumulus Oocyte Complex: Cumulus Cells Regulate Oocyte Growth and Maturation. J Mamm Ova Res. 2009;26:189-94; DOI:10.1274/jmor.26.189.

6. Vanikar A V., Mishra V V., Firoz A, Shah VR, Dave SD, Patel RD, Kanodia K V., Patel J V., Patel CN, Trivedi HL. Successful Generation of Donor Specific Hematopoietic Stem Cell Lines From Co-Cultured Bone Marrow With Human Embryonic Stem Cell Line: A New Methodology. Transplant Proc. 2007;39:658-61; DOI:10.1016/j.transproceed.2007.01.048.

7. Daniel MG, Pereira CF, Lemischka IR, Moore KA. Making a Hematopoietic Stem Cell. Trends Cell Biol. 2016;26:202-14; DOI:10.1016/j. tcb.2015.10.002.

8. Ye H, Li X, Zheng T, Liang X, Li J, Huang J, Pan Z, Zheng Y. The effect of the immune system on ovarian function and features of ovarian germline stem cells. Springerplus. 2016;5; DOI:10.1186/s40064-016-2390-3.

9. Hoheisel JD. Microarray technology: Beyond transcript profiling and genotype analysis. Nat Rev Genet. 2006;7:200-10; DOI:10.1038/ nrg1809.

10. Orkin SH, Zon LI. Hematopoiesis: An Evolving Paradigm for Stem Cell Biology. Cell. 2008;132:631-44; DOI:10.1016/j.cell.2008.01.025.

11. Jordan CT, McKearn JP, Lemischka IR. Cellular and developmental properties of fetal hematopoietic stem cells. Cell. 1990;61:953-63 DOI:10.1016/0092-8674(90)90061-I

12. Hwang SM, Sharma G, Verma R, Byun S, Rudra D, Im SH. Inflammation-induced Id2 promotes plasticity in regulatory T cells. Nat Commun. 2018;9:1-13; DOI:10.1038/s41467-018-07254-2.

13. Frias AB, Ji J, Buechel HM, Hand T, D'Cruz LM. Id protein function in adipose resident regulatory T cells. J Immunol. 2018;200.

14. Murre C. Helix-loop-helix proteins and lymphocyte development. Nat Immunol. 2005;6:1079-86; DOI:10.1038/ni1260.

15. Chermuła B, Brązert M, Jeseta M, Ożegowska K, Kocherova I, Jankowski M, Celichowski P, Sujka-Kordowska P, Konwerska A, Piotrowska-Kempisty H, Budna-Tukan J, Antosik P, Bukowska D, Machatkova M, Brussow KP, Skowroński MT, Pawelczyk L, Bruska M, Nowicki M, Kempisty B. Transcriptomic pattern of genes regulating protein response and status of mitochondrial activity are related to oocyte maturational competence-A transcriptomic study. Int J Mol Sci. 2019;20; DOI:10.3390/ ijms20092238.

16. Abhinand CS, Raju R, Soumya SJ, Arya PS, Sudhakaran PR. VEGF-A/VEGFR2 signaling network in endothelial cells relevant to angiogenesis. J Cell Commun Signal. 2016;10:347-54; DOI:10.1007/s12079-016-0352-8.

17. Salvi V, Vermi W, Gianello V, Lonardi S, Gagliostro V, Naldini A, Sozzan S, Bosisio D. Dendritic cell-derived VEGF-A plays a role in inflammatory angiogenesis of human secondary lymphoid organs and is driven by the coordinated activation of multiple transcription factors. Oncotarget. 2016;7:39267-9; DOI:10.18632/oncotarget.9684.

18. Trau H, Brännström M, ... TCJ-H, 2016 U. Prostaglandin E2 and vascular endothelial growth factor A mediate angiogenesis of human ovarian follicular endothelial cells. Hum Reprod. 2016;31:436-44.

19. Chermuła B, Brazert M, Jeseta M, Ożegowska K, Sujka-Kordowska $P$, Konwerska A, Bryja A, Kranc W, Jankowski M, Nawrocki MJ, Kocherova I, Celichowski P, Borowiec B, Popis M, Budna-Tukan J, Antosik P, Bukowska D, Brussow KP, Pawelczyk L, Bruska M, Zabel M, Nowicki M, Kempisty $B$. The unique mechanisms of cellular proliferation, migration and apoptosis are regulated through oocyte maturational development-A complete transcriptomic and histochemical study. Int J Mol Sci. 2019;20 DOI:10.3390/ijms20010084

20. Welt C, Schneyer A. Inhibin, Activin, and Follistatin in Ovarian Physiology. The Ovary, Elsevier; 2019, p. 95-105; D0I:10.1016/ b978-0-12-813209-8.00006-6.

21. Bernard DJ, Smith CL, Brûlé E. A Tale of Two Proteins: Betaglycan, IGSF1, and the Continuing Search for the Inhibin B Receptor. Trends Endocrinol Metab. 2020;31:37-45; DOI:10.1016/j.tem.2019.08.014.

22. Li D, Liu K, Li Z, Wang J, Wang X. miR-19a and miR-424 target TGFBR3 to promote epithelial-to-mesenchymal transition and migration of tongue squamous cell carcinoma cells. Cell Adh Migr. 2018;12:236-46; DOI:10. 1080/19336918.2017.1365992.
23. Batlle E, Massagué J. Transforming Growth Factor- $\beta$ Signaling in Immunity and Cancer. Immunity. 2019;50:924-40; DOI:10.1016/j. immuni.2019.03.024.

24. Fayad T, Lévesque V, Sirois J, Silversides DW, Lussier JG. Gene Expression Profiling of Differentially Expressed Genes in Granulosa Cells of Bovine Dominant Follicles Using Suppression Subtractive Hybridization1. Biol Reprod. 2004;70:523-33; DOI:10.1095/biolreprod.103.021709.

25. Assidi M, Dufort I, Ali A, Hamel M, Algriany O, Dielemann S, Sirard M-A. Identification of Potential Markers of Oocyte Competence Expressed in Bovine Cumulus Cells Matured with Follicle-Stimulating Hormone and/ or Phorbol Myristate Acetate In Vitro. Biol Reprod. 2008;79:209-22; DOI:10.1095/biolreprod.108.067686.

26. Si T, Lu Y, Li F, Jiang L, Pei R, Zhou JX. High expression of INHBA is an adverse prognostic factor for de novo acute myeloid leukemia. Leuk Lymphoma. 2018;59:114-20; DOI:10.1080/10428194.2017.1324157.

27. Lyu S, Jiang C, Xu R, Huang Y, Yan S. Inhba upregulation correlates with poorer prognosis in patients with esophageal squamous cell carcinoma. Cancer Manag Res. 2018;10:1586-96; DOI:10.2147/CMAR.S160186.

28. Su H, Chang J, Xu M, Sun R, Wang J. CDK6 overexpression resulted from microRNA-320d downregulation promotes cell proliferation in diffuse large B-cell lymphoma. Oncol Rep. 2019;42:321-7; DOI:10.3892/ or.2019.7144.

29. Li H, Zhang J, Xie Y. Elevated nuclear CDK6 is associated with an unfavorable prognosis in lung adenocarcinoma patients. Int J Clin Exp Pathol. 2017;10:9614-20.

30. Oqani RK, Lin T, Lee JE, Kim SY, Kang JW, Jin D Il. Effects of CDK inhibitors on the maturation, transcription, and MPF activity of porcine oocytes. Reprod Biol. 2017;17:320-6; DOI:10.1016/j.repbio.2017.09.003.

31. Basak A, Munschauer M, Lareau CA, Montbleau KE, Ulirsch JC, Hartigan CR, Schenone M, Lian J, Wang Y, Huang Y, Wu X, Gehrke L, Rice CM, An X, Christou HA, Mohandas N, Carr SA, Chen JJ, Orkin SH, Lander ES, Sankaran VG. Control of human hemoglobin switching by LIN28B-mediated regulation of BCL11A translation. Nat Genet. 2020;52:138-45; DOI:10.1038/s41588-019-0568-7.

32. Sankaran VG, Menne TF, Xu J, Akie TE, Lettre G, Van Handel B, Mikkola HKA, Hirschhorn JN, Cantor AB, Orkin SH. Human fetal hemoglobin expression is regulated by the developmental stage-specific repressor BCL11A. Science (80- ). 2008;322:1839-42; DOI:10.1126/ science.1165409.

33. Girón-Pérez DA, Vadillo E, Schnoor M, Santos-Argumedo L. Myo1e modulates the recruitment of B cells to inguinal lymph nodes. BioRxiv. 2019:668608; DOI:10.1101/668608.

34. Veerman K, Tardiveau C, Martins F, Coudert J, Girard JP. Single-Cell Analysis Reveals Heterogeneity of High Endothelial Venules and Different Regulation of Genes Controlling Lymphocyte Entry to Lymph Nodes. Cell Rep. 2019;26:3116-3131.e5; DOI:10.1016/j.celrep.2019.02.042.

35. Borys S, Brązert M, Jankowski M, Kocherova I, Ożegowska K, Celichowski P, Nawrocki MJ, Kranc W, Bryja A, Kulus M, Jeseta M, Pieńkowski W, Bręborowicz A, Bukowska D, Antosik P, Pawelczyk L, Skowroński MT Brüssow KP, Bruska M, Zabel M, Nowicki M, Kempisty B. Enzyme linked receptor protein signaling pathway is one of the ontology groups that are highly up-regulated in porcine Oocytes before in vitro maturation. J Biol Regul Homeost Agents. 2018;32:1089-103.

36. Xu Z, Zou L, Ma G, Wu X, Huang F, Feng T, Li S, Lin Q, He X, Liu Z, Cao X. Integrin $\beta 1$ is a critical effector in promoting metastasis and chemo-resistance of esophageal squamous cell carcinoma. Am J Cancer Res. 2017;7:531-42.

37. Burghardt RC, Johnson GA, Jaeger LA, Ka H, Garlow JE, Spencer TE, Bazer FW. Integrins and Extracellular Matrix Proteins at the Maternal-Fetal Interface in Domestic Animals. Cells Tissues Organs. 2002;172:202-17; DOI:10.1159/000066969.

38. Thiel G, Cibelli G. Regulation of life and death by the zinc finger transcription factor Egr-1. J Cell Physiol. 2002;193:287-92; DOI:10.1002/ jcp.10178.

39. Yuan S, Wen J, Cheng J, Shen W, Zhou S, Yan W, Shen L, Luo A, Wang S. Age-associated up-regulation of EGR1 promotes granulosa cell apoptosis during follicle atresia in mice through the NF- $\mathrm{KB}$ pathway. Cell Cycle. 2016;15:2895-905; DOI:10.1080/15384101.2016.1208873.

40. Veraguas D, Gallegos PF, Velasquez AE, Castro FO, Rodriguez-Alvarez L. FSH stimulation of anestrous cats improves oocyte quality and development of parthenogenetic embryos. Theriogenology. 2017;87:25-35; DOI:10.1016/j.theriogenology.2016.08.008.

41. Xu B, Zhang YW, Tong XH, Liu YS. Characterization of microRNA profile in human cumulus granulosa cells: Identification of microRNAs that reg ulate Notch signaling and are associated with PCOS. Mol Cell Endocrinol. 2015;404:26-36; DOI:10.1016/j.mce.2015.01.030.

42. Stefańska K, Józkowiak M, Antosik P, Bukowska D, Celichowski P, Bruska M, Nowicki M, Kempisty B, Zakova J, Machatkova M, Jeseta M. Genes reg- 
ulating programmed cell death are significantly upregulated in porcine immature oocytes. Med J Cell Biol. 2019; DOI:10.2478/acb-2019-0001.

43. Conde P, Morado S, Alvarez G, Smitz J, Gentile T, Cetica P. Effect of the hematopoietic growth factors erythropoietin and kit ligand on bovine oocyte in vitro maturation and developmental competence. Theriogenology. 2019;123:37-44; DOI:10.1016/j.theriogenology.2018.09.014.

44. Balounová J, Šplíchalová I, Dobešová M, Kolář M, Fišer K, Procházka J, Sedlacek R, Jurisicova A, Sung H ki, Koř́nek V, Alberich-Jorda M, Godin I, Filipp D. Toll-like receptor 2 expression on c-kit+ cells tracks the emergence of embryonic definitive hematopoietic progenitors. Nat Commun. 2019;10:1-14; DOI:10.1038/s41467-019-13150-0.

45. Li G, Yao L, Zhang J, Li X, Dang S, Zeng K, Zhou Y, Gao F. Tumor-suppressive microRNA-34a inhibits breast cancer cell migration and invasion via targeting oncogenic TPD52. Tumor Biol. 2016;37:7481-91; DOI:10.1007/s13277-015-4623-4.

46. Kumamoto T, Seki N, Mataki H, Mizuno K, Kamikawaji K, Samukawa T, Koshizuka K, Goto Y, Inoue H. Regulation of TPD52 by antitumor microRNA-218 suppresses cancer cell migration and invasion in lung squamous cell carcinoma. Int J Oncol. 2016;49:1870-80; DOI:10.3892/ ijo.2016.3690. 